\title{
Interpolated task characteristics and interference in short-term memory
}

\author{
CHARLES P. BIRD and GEORGE E. WEAVER \\ Florida State University, Tallahassee, Florida 32306
}

\begin{abstract}
The effect of variations in interpolated activity on word recall was examined. Two levels of interpolated task difficulty were factorially combined with three levels of similarity of interpolated items to critical items. The interpolated activities involved either verbal (number or word classification) or nonverbal (tone classification) stimuli. Results indicated that interpolated task difficulty was an important factor influencing word recall at all levels of similarity. In addition, the number and word classification tasks did not differ reliably in their effect on recall, although both resulted in poor recall relative to that following a tone classification task.
\end{abstract}

Several recent studies have attempted to account for information loss in the Brown-Peterson paradigm (e.g., Reitman, 1971, 1974; Shiffrin, 1973; Watkins, Watkins, Craik, \& Mazurik, 1973). These studies typically have been directed toward determining whether information is lost from some short-term store as a function of the passage of time ("decay") or only as a function of displacement by similar information.

The results of several of these studies appear to be quite consistent with the displacement hypothesis. Both Reitman (1971) and Shiffrin (1973) found no significant decrease in retention of words over retention intervals up to $40 \mathrm{sec}$ if the interval was filled with activity on a nonverbal detection task. It was argued that while the detection task itself did not lead to displacement of items from short-term storage, it did prevent the maintenance of items during the interval via rehearsal. Given this assumption, these data were interpreted as indicating that information is not lost during a retention interval, even in the absence of rehearsal, except through displacement by similar cognitive activity.

However, other data suggest that even a nonverbal task can produce retention losses in the Brown-Peterson situation if the task is sufficiently difficult. Thus, both Watkins et al. (1973) and Reitman (1974) obtained reliable decreases in word recall over retention intervals of 1 to $20 \mathrm{sec}$ when a very difficult tone detection or classification task was employed. Although there is little empirical evidence available, the importance of difficulty seems not to be limited to situations involving nonverbal interpolated tasks. For example, Posner and Konick (1966) found that increasing the difficulty of an interpolated arithmetic task led to poorer performance in the recall of letters or of other digit stimuli. However, in this latter study, there was no indication of an effect of similarity, i.e., arithmetic had no more effect on digit recall than on letter recall.

While various data suggest that both task difficulty and task similarity influence information loss, there have been few attempts to systematically investigate their influence. Such data would seem important in the context of the suggestion that the effect of interpolated task difficulty (whether verbal or nonverbal) is through interference with general processing (e.g., rehearsal) of critical items. In contrast, similarity of the interpolated task presumably produces interference due to displacement of critical items from a short-term store (Reitman, 1974) or through a reduction in the discriminability of critical and interpolated items at the time of recall (Watkins et al., 1973). The present study was an attempt to vary both task difficulty and the similarity of interpolated items to critical items when the critical items were words. It was hoped that data from this study might permit a more comprehensive view of the relative importance of task difficulty when both verbal and nonverbal distractor tasks were compared in the same study.

The design followed the general Brown-Peterson procedure and involved the factorial combination of two levels of interpolated task difficulty and three levels of similarity of interpolated items to the critical items. Thus, one of the distractor tasks involved tone classification, one involved digit classification, and the third required a verbal response to word stimuli. While the selection of tasks was arbitrary, a nonverbal task and two verbal tasks were included, the latter tasks differing in degree of similarity of items and processing activity to the primary task of word recall. For each type of task, two levels of difficulty were established based on the number of decision alternatives involved in correctly performing the task.

\section{METHOD}

\section{Design and Materials}

The design was a 3 by 2 factorial in which the factors were similarity of interpolated items to critical items and interpolated task difficulty. Specifically, subjects were required to classify either two tones or four tones; to classify numbers as odd or even, or as both odd or even and above or below some reference number; and to repeat words or to classify words as nouns or verbs. 
Table 1

Mean Word Recall as a Function of Interpolated Activity

\begin{tabular}{llrcr}
\hline & & \multicolumn{3}{c}{ Interpolated Task } \\
\cline { 3 - 5 } Difficulty & & Tones & Numbers & Words \\
\hline \multirow{2}{*}{ Easy } & Mean & 45.67 & 32.67 & 30.67 \\
& SD & 5.02 & 6.41 & 3.92 \\
\multirow{2}{*}{ Difficult } & Mean & 38.40 & 23.53 & 22.73 \\
& SD & 6.53 & 6.58 & 5.50 \\
\hline
\end{tabular}

Five critical items were presented on each trial, followed by $20 \mathrm{sec}$ of interpolated activity and a test of retention. A total of 10 trials were administered to each subject. The critical items were single-syllable nouns with mean frequencies for the five items on each trial ranging from 58.8 to 60.4 (Kučera \& Francis, 1967). Any obvious formal or semantic similarity among items presented on a given trial was eliminated.

Each set of five words was printed in block letters on a single $2 \times 2$ in. slide in the form of a verticle array; each word shifted one letter to the right of the word above. These items, as well as various visual cues, were presented by a Carousel projector and projected on a screen about $1 \mathrm{~m}$ in front of the subject. Presentation of slides was controlled by a Hunter timer, and the recall intervals were timed with a stopwatch. Items for all interpolated activities were presented by a tape recorder, with 10 item s being presented during each retention interval.

The tasks involving tones required subjects to classify tones from a piano according to their relative position on the musical scale. The easy tone task involved two tones-middle $\mathrm{C}$ and $\mathrm{G}$ above middle $\mathrm{C}$-while the difficult task involved four tones-middle $\mathrm{C}, \mathrm{E}, \mathrm{G}$, and $\mathrm{C}$ above middle $\mathrm{C}$. The subject simply responded to each tone as it occurred by pressing the appropriate button on a response panel. The panel had either two buttons or four buttons, depending on the condition, and responses were monitored by the experimenter. The buttons on the subject's panel were labeled "one," "two." etc., with "one" corresponding to the lowest tone.

The easy digit task involved classifying random two-digit numbers as odd or even, while the difficult task required subjects to classify numbers as odd or even and above or below a reference number (37). The same response panels as employed for the tone task were used, with appropriate labels provided for the buttons ("odd" and "even" for the easy task, "below-odd," "below-even," etc., for the difficult task).

The interpolated tasks involving words employed nouns and verbs and required overt verbal responses from the subject. The easy task involved repeating each word as it occurred; the difficult task involved classifying each item as a noun or a verb by saying "noun" or "verb" aloud. Formal and semantic similarity to critical items was minimized.

It was intended that the easy tone and number tasks involve a decision between two alternatives, while the corresponding difficult tasks involve four alternatives. On the other hand, the easy word task required no decision, and the difficult word task involved a decision between two alternatives. Thus, in terms of decision alternatives, the word tasks should have been less difficult than corresponding tasks in the tone and number conditions.

\section{Procedure}

Instructions were read by the subject and 1 min of practice was provided on the interpolated activities $(2 \mathrm{~min}$ were provided on the difficult tone task). Each trial began with a ready signal (a 2.5 -sec presentation of a slide with three asterisks), followed by the five critical items, also presented for $2.5 \mathrm{sec}$. While the critical items were on the screen, the subject read each word aloud. Another asterisk slide followed the critical items, and it remained on the screen throughout the retention interval. Beginning immediately after the offset of the critical items, subjects worked on the appropriate interpolated activity, with the 10 items being presented at a $2-\mathrm{sec}$ rate. At the end of the retention interval, a slide with three question marks signaled the beginning of a $20-\mathrm{sec}$ recall interval. At the end of the recall interval, the next trial began immediately. Recall was oral and to provide a scoring check, the entire session was tape recorded.

\section{Subjects}

Fifteen subjects participated in each of the six conditions, making a total of 90 subjects. All subjects were tested individually and were obtained from the introductory psychology classes at Florida State University.

\section{RESULTS AND DISCUSSION}

Table 1 presents the mean recall for all 10 trials combined. It is clear that performance decreased as a function of difficulty at all levels of task similarity. It is also of interest to note that the difference in the means for the easy and difficult tasks is extremely consistent at the various levels of task similarity. In addition, although there is very little difference in recall for the conditions involving the number and word tasks, both these tasks produced poorer recall than the tone tasks.

The data was analyzed as a 3 by 2 factorial in which the factors were task similarity and task difficulty. Significant main effects were obtained for both similarity and difficulty, $F(2,84)=65.30$ and $F(1,84)=$ $44.83, \mathrm{p}<.001$ in each case, but the interaction of similarity and difficulty was not reliable, $F(2,84)<1$. As is evident in Table 1 , the absence of a significant interaction indicates that the effects of task difficulty were comparable at all levels of task similarity. A Newman-Keuls analysis calculated for the main effect of similarity revealed that word recall for the number and word conditions did not differ reliably $(p>.10)$, but both of these activities produced significantly poorer recall than the tone activity ( $\mathrm{p}<.001$ in each case).

In summary, these data are largely consistent with the arguments advanced by Watkins et al. (1973) and Reitman (1974). Clearly, a nonverbal task can interfere with word recall provided it is sufficiently difficult. It also appears that task difficulty is an important factor to be considered when the interpolated activity is verbal. With regard to similarity, it is apparent that verbal tasks are more interfering than nonverbal tasks. The failure to obtain a difference between the number and word tasks is consistent with the Posner and Konick (1966) finding that an arithmetic distractor task had no more effect on digit recall than on letter recall. Thus, although the precise reasons for the difficulty and similarity effects remain to be determined, the present study has provided strong evidence to indicate that both factors must be considered in any attempt to explain information loss in the Brown-Peterson paradigm. 


\section{REFERENCES}

Kučera, H., \& Francis, W. N. Computational analysis of present day American English. Providence: Brown University Press, 1967.

Posner, M. I., \& Konick, A. F. On the role of interference in short-term retention. Journal of Experimental Psychology, 1966, 72, 221-231.

Reitman, J. S. Mechanisms of forgetting in short-term memory. Cognitive Psy chology, 1971, 2, 185-195.
Reitman, J. S. Without surreptitious rehearsal, information in short-term memory decays. Journal of Verbal Learning and Verbal Behavior, 1974, 13, 365-377.

Shiffrin, R. M. Information persistence in short-term memory. Journal of Experimental Psy chology, 1973, 100, 39-49.

Watkins, M. J., Watkins, O. C., Craik, F. I. M., \& Mazurik, G Effect of nonverbal distraction of short-term storage. Journal of Experimental Psy cholngy, 1973, 101, 296-300.

(Received for publication March 24, 1974.) 